\title{
Professor Leonid I. Manevitch
}

\author{
Igor V. Andrianov - Oleg V. Gendelman - Margarita A. Kovaleva • \\ Yuri V. Mikhlin • Valery N. Pilipchuk
}

Published online: 4 November 2020

(C) Springer Nature B.V. 2020

\section{Professor Leonid I. Manevitch}

02.04.1938-20.08.2020

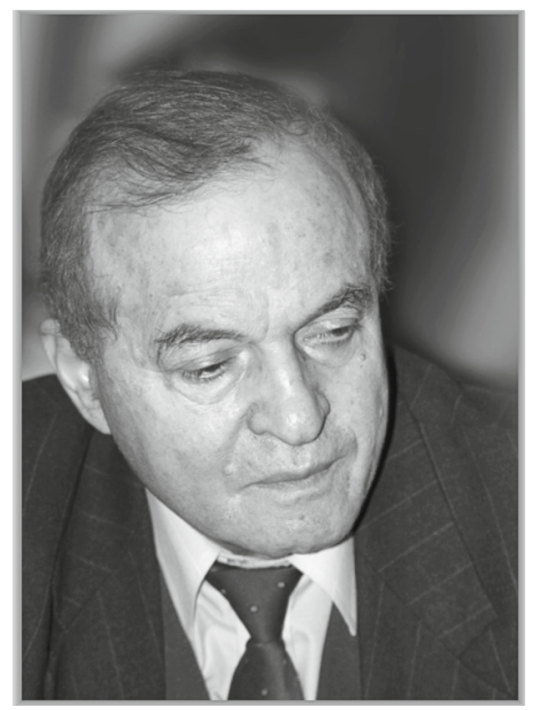

I. V. Andrianov

RWTH Aachen University, Aachen, Germany

O. V. Gendelman

Technion - Israel Institute of Technology, Haifa, Israel

M. A. Kovaleva

N.N. Semenov Federal Research Center for Chemical

Physics, Russian Academy of Science, Moscow, Russia
The scientific community lost an outstanding and original scientist, prominent creative and fruitful in the field of Nonlinear Dynamics, Mechanics of Solids, Condensed Matter Physics. After severe illness, Professor Leonid I. Manevitch passed away on August 20, 2020, at the age 82. He worked on scientific problems and proposed new ideas until the very last days of his life.

Leonid Manevitch was born on April 2, 1938, in Mogilev (USSR, currently Belarus). His father and mother were physicians. During the Second World War, the family moved out and, after the war, settled in Dnepropetrovsk (now Dnipro). Leonid Manevitch attended his graduate studies at the Faculty of Physics and Mathematics of Dnepropetrovsk State University (DSU) in 1954-1959. After graduating from the DSU summa cum laude, he worked as an aerospace stress engineer and a head of the Stress Analysis Team in the Yangel Yuzhnoye State Design Office (1959-1964), and at the same time, he studied in extramural doctoral study at DSU. He got his PhD degree with a thesis on

\footnotetext{
Y. V. Mikhlin ( $₫)$

National Technical University «Kharkiv Polytechnic

Institute», Kharkiv, Ukraine

e-mail: Yuriy.Mikhlin@khpi.edu.ua;

Yuri.Mikhlin@gmail.com

V. N. Pilipchuk

Wayne State University, Detroit, USA
} 
stability of shells (under supervision by Prof. Yu. A. Shevlyakov) in 1961. From 1964 until 1976, he worked at DSU as an Associate Professor and then as a Full Professor in the Department of Applied Theory of Elasticity led by Academician V.I. Mossakovsky. In 1970, he received the degree of Doctor of Sciences for his thesis on asymptotic and group methods in the mechanics of deformable solids, and later on, in 1973, he received the title of Professor. Since 1976, after moving to Moscow, he worked by special invitation of Nobel Prize laureate N.N. Semenov as a senior research fellow, then as the head of the Physics and Mechanics of Polymers Division of the Institute of Chemical Physics of the USSR (later Russian) Academy of Sciences, and as a senior research fellow during the last years of his life. This Laboratory under his leadership became one of the well-known research teams in the field of solid polymer physics and nonlinear dynamics of molecular systems, which actively cooperates with research centers from different countries. Since 1984, he also worked as a Professor in the Department of Polymer Physics at the Moscow Institute of Physics and Technology.

Prof. Manevitch's scientific activity was related to many areas of mechanics of deformable bodies, nonlinear dynamics and condensed matter physics. The breadth of his investigations is striking. He could formulate universal concepts and ideas for different mathematical, mechanical, and physical problems.

In particular, he obtained important and fruitful results in the theory of anisotropic and reinforced plates and shells, in the theory of buckling and postbuckling behavior of elastic structures. He obtained important results in the asymptotic analysis of elasticity theory for anisotropic media, including problems of deformation and destruction of highly anisotropic composites.

He has made significant contributions to the theory of nonlinear normal vibration modes of essentially nonlinear systems, including vibrating systems with multiple equilibrium positions and/or stiff barriers, where energy localization and interaction of vibration modes are observed. L.I. Manevitch with his collaborators developed the method known as "complexification-averaging" to describe nonstationary processes in a multitude of physical contexts. In his last years of activity, he proposed and developed the principally new paradigm in nonstationary dynamics, namely a concept of Limiting Phase Trajectories which represent the fundamental essentially nonstationary process, which is responsible for the energy exchange between some clusters of particles.

Together with the analytical description of dislocations in polymer crystals obtained by Prof. L.I. Manevitch and his co-workers, the molecular dynamics simulation of both edge and screw dislocations in such crystals was first carried out; the mobility of dislocations was studied. He developed the theory of structural defects and structural transitions at the atomic level in molecular and polymer crystals, studied the transport and localization of energy in mechanical systems, polymer chains, and nanotubes. A continuum model of a low-frequency localized mode in polymer glasses was developed; estimates of their yield strength were first obtained. A theory of topological solitons in molecular chains with a nondegenerate bi-stable interaction potential was devised. Localized excitations in the graphene layer and the carbon nanotube were first revealed in his works.

The nonlinear lattices with a complex structure were the focus of his interests for a long time. Key results were obtained, in particular, in the study of torsion solitons and the breather-like excitations in the polymer chains. Important developments in the plastic deformation theory and in phase transitions of polymer crystals were achieved. A solution of the problem of the supersonic propagation of chemical reactions in solid media was found and presented in a series of publications.

Professor L.I. Manevitch was an active participant of many Russian and International symposia, conferences and congresses. As a guest speaker he repeatedly appeared at seminars of famous universities in the USA, Europe and Israel (Harvard University, Massachusets Institute of Technology, Brown University, University of Illinois at Urbana-Champaign, Sapienza University of Rome, Delft University of Technology, Lodz University of Technology, Universities of Groningen, Ulm, Freiburg, Technion-Israel Institute of Technology, etc.). His scientific results are presented in 20 monographs and in more than 350 publications.

Leonid Manevitch was not only an excellent researcher, but also a great mentor. Under his guidance $35 \mathrm{PhDs}$ were completed, and 12 of his disciples became Doctors of Science (the highest academic degree in the former Soviet Union and post-Soviet countries). His boundless enthusiasm and outstanding 
pedagogical talent allowed him to create a large scientific circle of affiliates and followers. The collection of articles dedicated to the 80th anniversary of Prof. Manevitch [1] allows to assess the scale and scientific level of this school. This book also contains a detailed overview of his scientific activities.

1. Andrianov, I.V., Manevich, A.I., Mikhlin, Yu.V., Gendelman, O.V. (Eds.): Problems of Nonlinear Mechanics and Physics of Materials. Advanced Structured Materials, vol 94. Springer Nature, Cham (2019).

Professor Giuseppe Rega (Sapienza University of Rome) wrote: "Professor Leonid Manevitch was a giant of nonlinear dynamics. Besides mentoring a great number of Russian scientists who are now mature or younger professors in prestigious academic institutions, since the mid-1990s, he was likely the scholar who, covering a wide area of knowledge beyond mechanics, more strongly affected the development of a meaningful number of western scientists active in nonlinear dynamics in mechanics. I was struck by Professor Manevitch's strong personality at the 2003 IUTAM Symposium in Rome, and then in many other occasions. Yet, having the privilege to contribute a paper to a Conference in his honor at the RAS Semenov Institute, Moscow 2008, and to host him at several scientific events in Italy, the warmth of his personality became fully apparent, too. Professor Manevitch will be greatly missed by the worldwide community of nonlinear dynamics in mechanics from both the scientific and the human side."

Professor Jan Awrejcewicz (Łódź University of Technology) wrote: "I met Professor L.I. Manevitch at one of the first DSTA conferences organized in Łódź, Poland. Then I had the opportunity to talk to him frequently, following his interdisciplinary developments in nonlinear mechanics, physics, chemistry and applied mathematics. I had the pleasure of working with him while writing two co-authoring monographs. His life was characterized by a great scientific passion and he certainly developed several new research directions in nonlinear science, including (i) asymptotic interpretation of wave-particle duality as an efficient analytical tool for solving both linear and nonlinear nonstationary dynamical problems of classical/quantum mechanics; (ii) nonlinear resonances interaction and energy localization in carbon nanotubes; (iii) modeling and analysis of van der Waals interactions with regard to the nanotube sizes and crystal symmetries; (iv) long-time resonant energy exchange in carbon nanotubes from the optical perspective; (v) recently developed the concept of limiting phase trajectories providing a unified description of resonant energy transport in a wide range of classical and quantum dynamical systems; (vi) new coarse-grained models and dynamic properties of DNA molecules."

Professor Vladimir Babitsky (Loughborough University) wrote: "It is hard to believe that such a creative and productive life was interrupted so suddenly. Leonid was always in constant development, working productively until the last days. His unusually broad erudition, the highest professionalism and an original vision of problems allowed him to generate constantly the promising ideas and concepts that have been reflected in his numerous and highly qualified publications receiving wide international recognition. Bright pedagogical talent allowed Leonid to educate a lot of talented students and followers. The loss of such an outstanding specialist and charming person is irreplaceable. His remarkable human qualities: tact, intelligence, modesty will remain for a long time in the memory of everyone who knew him."

Professor Francesco Pellicano (Modena University) wrote: "I met Leonid in Kharkov in 2004 during the Nonlinear Dynamics Conference. I was honored to host him in 2011 in Modena for a seminar on irreversible energy transfer and localization; since then we have had a continuous scientific cooperation. From Leonid I learned a deep passion for research. I will never forget this great scientist and great man, his kindness and his enthusiasm."

Professor Alexander F. Vakakis (University of Illinois at Urbana-Champaign) wrote: "It is with great sadness that I learned of the recent death of Professor Leonid Isaakovich Manevitch. Throughout his life he was an admired scholar, a mentor to generations of nonlinear dynamicists, a valued colleague, and a wonderful and warm friend. He had a true passion for research and discovery, establishing his own school of nonlinear dynamics and chemical physics in Moscow. In fact, he was working on research until the very end-just days before his passing. I am sure that his many successful students and collaborators throughout the world will keep his legacy alive in the years to come. His friends, colleagues, and our entire community bid a heartfelt 
farewell to this great scientist. All of us will miss him dearly."

We, disciples and collaborators of Professor Manevitch, remember our study and long-time contacts with him as the happiest time of our life due to the genuine enthusiasm during these interactions and due to his bright, clear and profound ideas. L.I. Manevitch provided great influence on the work and lives of all his students and collaborators. We remember his constant attention and support in our study and work. We and our colleagues remember his love for research, true understanding of scientific problems, and intelligence.

H. Poincaré wrote: "Thought is but a lightning in the middle of a long night. But it is that lightning that is everything". L.I. Manevitch lived with lightning. An enviable fate!

\section{List of selected books of Professor Leonid I. Manevitch (in reverse chronological order)}

1. Manevitch L.I., Kovaleva A.S., Smirnov V.V., Starosvetsky Yu.: Nonstationary Resonant Dynamics of Oscillatory Chains and Nanostructures. Springer Nature, Singapore (2017)

2. Manevitch L.I., Gendelman O.V.: Tractable Models of Solid Mechanics. Formulation, Analysis and Interpretation. SpringerVerlag, Berlin, Heidelberg, (2011)

3. Manevitch L.I., Smirnov V.V.: Solitons in Macromolecular Systems. Nova Science Publishers, New York (2008)

4. Manevich A.I., Manevitch L.I.: The Mechanics of Nonlinear Systems with Internal Resonances. Imperial College Press, London (2005)

5. Andrianov I.V., Awrejcewicz J., Manevitch L.I.: Asymptotical Mechanics of Thin-Walled Structures. Springer, Berlin, Heidelberg, New York (2004)
6. Andrianov I.V., Barantsev R.G., Manevitch L.I.: Asymptotical Mathematics and Synergetics, URSS, Moscow (2004) (in Russian)

7. Manevitch L.I., Andrianov I.V., Oshmyan V.G.: Mechanics of Periodically. Heterogeneous Structures. Springer, Berlin, Heidelberg, New York (2002)

8. Andrianov I.V., Manevitch L.I.: Asymptotology. Ideas, Methods, and Applications. Kluwer Academic Publishers, Dordrecht, Boston, London (2002)

9. Awrejcewicz J., Andrianov I., Manevitch L.: Asymptotic Approaches in Nonlinear Dynamics: New Trends and Applications. SpringerVerlag, Berlin, Heidelberg (1998)

10. Vakakis A.F., Manevitch L.I., Mikhlin Yu.V., Pilipchuk V.N., Zevin A.A.: Normal Modes and Localization in Nonlinear Systems. Wiley, New York (1996)

11. Manevitch L.I., Mikhlin Yu.V., Pilipchuk V.N.: The Method of Normal Oscillations for Essentially Nonlinear Systems. Nauka, Moscow (1989) (in Russian)

12. Andrianov I.V., Lesnichaya V.A., Manevitch L.I.: The Averaging Method in Statics and Dynamics of Ribbed Shells. Nauka, Moscow (1985) (in Russian)

13. Manevitch L.I., Pavlenko A.V., Koblik S.G.: Asymptotic Methods in the Theory of Orthotropic Solids. Vysshaya Shkola (High School), Kiev (1982) (in Russian)

14. Mossakovskii V.I., Manevitch L.I., Miltzin A.M.: Modeling of Strength of Thin Shells. Naukova Dumka, Kiev (1977) (in Russian)

Publisher's Note Springer Nature remains neutral with regard to jurisdictional claims in published maps and institutional affiliations. 